\title{
Nuclear Fusion from Crack-Generated Particle Acceleration ${ }^{1}$
}

\author{
Frederick J. Mayer, ${ }^{2}$ John S. King, ${ }^{3}$ and John R. Reitz ${ }^{4}$
}

\section{INTRODUCTION}

The apparent observation of $d-d$ nuclear fusion in electrochemical cells ${ }^{(1,2)}$ is being interpreted, by some researchers, to be the result of the creation of "massive" electrons ("quasi-particles") or some other exotic mechanisms, in certain deuterium-infused metallic lattices. An important inconsistency has been noted between the neutron generation rates, on the one hand, and the excess electrochemical cell heating on the other. However, the usual method of producing d-d neutrons by accelerating a deuteron in an electrostatic field and colliding with another deuteron has not been ruled out, or even mentioned, as far as we know. ${ }^{5}$ Because of these inconsistencies and because a "classical" interpretation of the results has not been ruled out, it may be premature to invoke either "new" particles or "exotic" nuclear reactions to explain the observations. Specifically, in this paper, we propose a "classical" interpretation for the $\mathrm{d}-\mathrm{d}$ neutron production in the so-called "cold" fusion experiments.

It has been recognized for some time that highenergy processes can accompany the fracture of bonds in crystalline materials. ${ }^{(3,4)}$ Transient electrostatic potentials as high as $200 \mathrm{keV}$ have been measured in fracture or crack formations primarily in salt crystals stressed by various agents. Indeed, Klyuev et al. ${ }^{(5)}$ have reported the generation of $\mathrm{d}-\mathrm{d}$ neutrons during the shock wave-

' Presented at the Workshop on Cold Fusion Phenomena, Sante Fe, New Mexico, May 23-25, 1989.

${ }^{2}$ FJM Associates, 1417 Dicken Dr., Ann Arbor, Michigan 48103.

${ }^{3}$ Department of Nuclear Engineering, University of Michigan, Ann Arbor, Michigan 48109.

${ }^{4} 2260$ Chaucer Court, Ann Arbor, Michigan 48103.

${ }^{5}$ During preparation of this paper, we discovered that such a mechanism had been suggested independently by $\mathrm{H}$. Furth et al. at the Baltimore meeting of the American Physical Society, May 1, 1989. initiated "fracturing" of lithium deuteride. It seems reasonable, then, to suggest that the strongly stressed metals used in the "cold" fusion cells experience a similar rapid buildup of stress fractures, electrostatic charge imbalance, and consequent acceleration of some of the deuterons to quite high energies. The energetic deuterons, in turn, generate d-d neutrons and tritium as by-products. This is a quite familiar nuclear reaction generation mechanism, however, in an unfamiliar place--inside of a metal.

\section{THE ELECTROSTATIC FIELD AND NEUTRON GENERATION}

That the electric fields can be quite high is easy to show. Consider two opposing crystal planes, (similar to the parallel plate capacitor of Fig. 1) to be oppositely charged with $\eta<1$ electrons per square lattice constant ( $\sim 4$ angstroms), and separated by a distance $D$ centimeters. The voltage across the gap is $V=10^{9} \mathrm{\eta D}$ volts. So, for example, if $\eta=0.1$ and $D=10^{-5} \mathrm{~cm}$, then

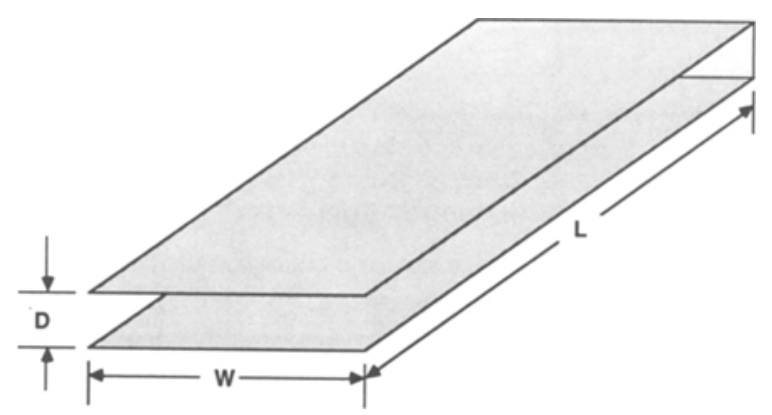

Fig. 1. Schematic of the idealized crack - a parallel plane capacitor that is shorted at one end. 
the voltage across the gap is $V=1 \mathrm{keV}$, or if $D=$ $10^{-4} \mathrm{~cm}$, then the voltage across the gap is $V=10 \mathrm{keV}$. Therefore, if during the time that the crystal planes separate, the charge is maintained, then deuteron acceleration can lead to energies of this order. Larger separations, of course, produce higher electric fields and deuteron energies. The higher deuteron energies are sufficient to produce on the order of a few neutrons per second per deuteron (see Fig. 2 which has assumed a deuterium/ palladium loading ratio of 1 and the d-d cross-section taken from Ref. 6). ${ }^{6}$

Given only about $10^{3}-10^{4}$ accelerated deuterons, the expected neutron generation rates are then of the order of those reported in references. ${ }^{(1,2)}$ Substantially higher reaction rates are produced at larger separations.

\section{THE RC DISCHARGE TIME}

Because the separation distance, $D$, is fairly small, the time required to maintain the surface charge is also fairly small. We might expect the surfaces to be receding from one another at roughly the speed of sound in the metal. If this is the case, then times of the order of something less than a nanosecond, depending upon the spacing, are required to maintain the surface charge.

Because of the importance of the time constants associated with the discharge of surface charge in the "cracked" lattice idea, we have estimated the times with a simple model "crack." Figure 1 shows the simple

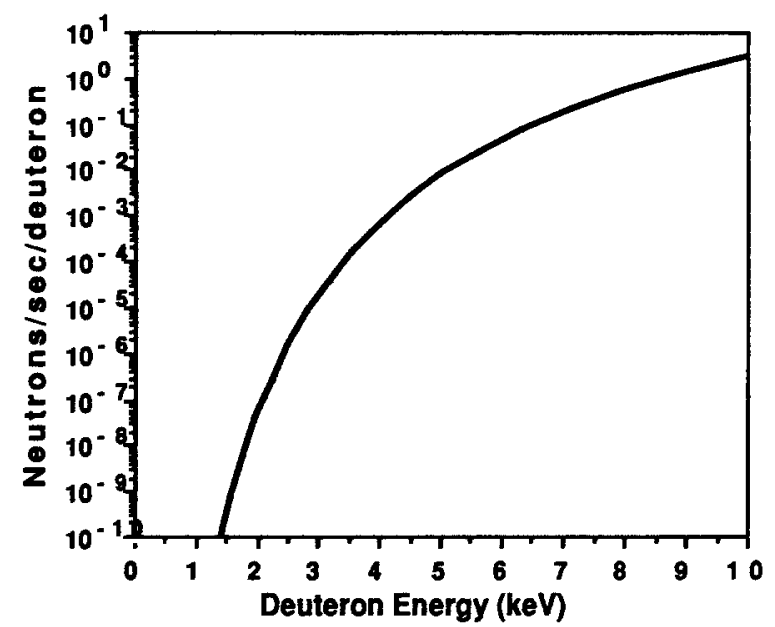

Fig. 2. The number of neutrons per second generated by a deuteron with velocity corresponding to energy $E_{d}$.

'See Ref. 6 (p. 268) for d-d cross-sections vs. energy. geometry of the cracked region as a capacitor shorted at one end. The relevant electrical quantities for such a capacitor can be found in, for example, Ref. 6. The circuit capacitance and inductance are given by,

$$
C_{c}=\epsilon_{o} W L / D \quad L_{c}=\left(\mu_{o} / 2 \pi\right) D L / W
$$

where the dimensions are as shown in Fig. 1, and the other symbols have their usual meanings. We take the vacuum permittivity and permeability in the gap between the charged planes. The circuit resistance is taken to be,

$$
R_{c}=\rho_{c} L / W \delta
$$

where $\delta$ is the effective layer thickness of the conduction path, and $\rho_{c}$ is the resistivity of the conduction path. The following are the various time constants ( $c$ is the speed of light):

$$
\begin{aligned}
\tau_{L C} & =\left(L_{c} C_{c}\right)^{1 / 2}=(2 \pi)^{-1 / 2}(L / c)^{1 / 2} \\
\tau_{R C} & =R_{c} C_{c}=\epsilon_{o} \rho_{c} L^{2} / D \delta \\
\rho_{L / R} & =L_{c} / R_{c}=\left(\mu_{o} / 2 \pi\right) D \delta / \rho_{c}
\end{aligned}
$$

The times scale, $\tau_{\text {acc }}$, for the deuteron acceleration is essentially the gap formation time. This is roughly the gap width divided by the speed of sound, $c_{s}$, which we take to be $2000 \mathrm{~m} / \mathrm{s}$. For $D=0.1 \mu \mathrm{m}, \tau_{\text {acc }}=50 \mathrm{ps}$ and for $D=1.0 \mu \mathrm{m}, \tau_{\text {acc }}=500 \mathrm{ps} \mathrm{cm}$, then $\tau_{\text {acc }}=$ 500 ps. We take the resistivity to be, $\rho_{c}=4 \times 10^{-7}$ ohm-m, ${ }^{(7)}$ and make a crude assumption for $\delta=100$ angstroms. Notice that the width $W$, cancels out in the time constants, so we can evaluate the time constants as a function of $L$ and $D$. By far, the largest of the three is $\tau_{R C}$ which is plotted in Fig. 3. From Fig. 3, we find that the time constant is larger than the acceleration time if $L>100$ microns for a 0.1 micron gap, and $L>1000$ microns for a 1-micron gap. Of course, there are numerous effects that could lengthen the discharge time constant substantially. Some examples are: the discharge path could be rather circuitous-increases $L$, a rather thinner conduction path thickness-increases resistance, structural defects near the crack may lead to increased resistivity, additional microcracking near the crack leading to increased resistivity, and probably others. It seems that most processes will tend to increase the discharge time, allowing the charge imbalance to persist for a longer period of time.

As mentioned before, that cracking does occur, at least in some situations, is well documented. Jamieson et al. ${ }^{\left({ }^{8}\right)}$ shows photomicrographs of large cracks observed in heavily hydrogen-loaded palladium. Furthermore, neutron production at a similarly low level has been observed by Klyuev et al. ${ }^{(5)}$ in experiments that 


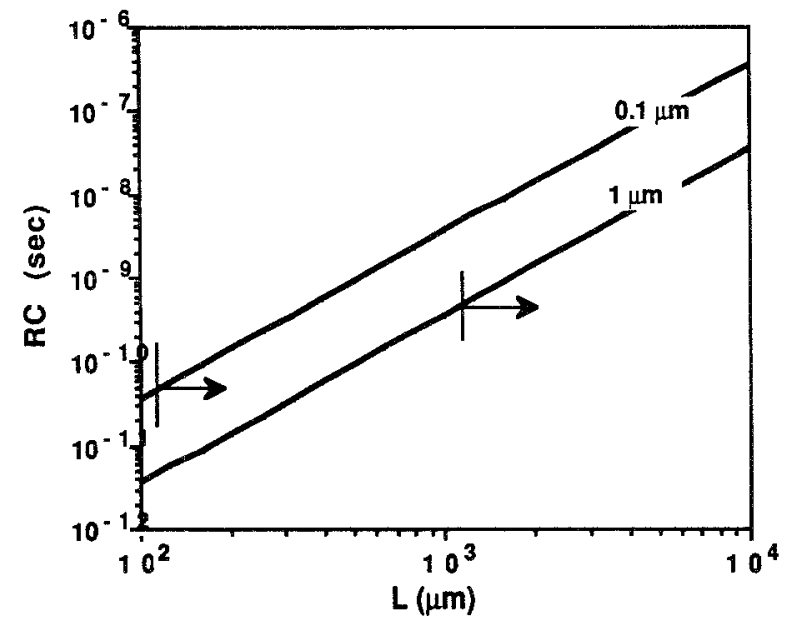

Fig. 3. A plot of the $\mathrm{RC}$ time constant as a function of the length, $L$, to the capacitor's shorted end, for crack separation distances of 0.1 and $1.0 \mu \mathrm{m}$. Crack lengths larger than those indicated by the arrows have sufficient time to fully accelerate the deuterons.

induced fracturing in a LiD crystal with a gas-gun driven projectile. In contrast to the deuterium-loaded metal experiments, however, the LiD is a dielectric, allowing charge imbalance to persist for much longer times.

Because there is an entire literature involving the generation of energetic particles and radiation initiated by fracturing a material, the reader is encouraged to look at the work of J. T. Dickinson of Washington State University and V. A. Klyuev in the Soviet Union. Particularly relevant to our crack proposal is the paper by Ryan et al. ${ }^{(9)}$ at this workshop. The material cracking/neutron generation correlation may also be consistent with the observations of $\mathrm{d}-\mathrm{d}$ neutrons released in bursts as seen by Menlove et al.,$^{(10)}$ also in a paper presented at this workshop.

If cracking is responsible for neutron generation, then it might be possible to maximize the effect by using a projectile impact spall-type experiment on a heavily deuterium-loaded palladium plate. This experiment $t^{(11)}$ is already being considered, and other geometries for optimizing the effect could also be investigated.

\section{STORED ELECTROSTATIC ENERGY}

Because of the usefulness of energy accounting in the studies of "cold" fusion experiments, a comment is perhaps in order about the amount of energy which could be stored and then released in the cracking process. The sequence is: electrical to mechanical energy storage by the lattice, then the release is from mechanical to electrical, and finally, to thermal energy. The amount of energy stored in the crack electrostatic fields, which may be rather rapidly discharged as an "arc" is easily calculated with our simple capacitor model. The charged planes separated by a distance $D$, have a stored energy of about $E=10^{5} \eta^{2} D / 2$ joules $/ \mathrm{cm}^{2}$. Again taking $\eta=0.1$ and $D=10^{-4} \mathrm{~cm}$, we have $E=0.05$ joules $/ \mathrm{cm}^{2}$. If crack planes were created and then the electrostatic energy discharged at a rate of tens of square centimeters per second, then watts of electrical power would be discharged. This could be important to understanding the energy partitioning in the electrochemical cells.

\section{SUMMARY}

In summary, the high-voltages necessary to accelerate deuterons to energies sufficient to produce modest numbers $\left(10^{4}-10^{5} / \mathrm{sec}\right)$ of $\mathrm{d}-\mathrm{d}$ neutrons appears to be possible as a result of cracking or "fracture" of the metal lattice in the "cold" fusion experiments.

This mechanism requires neither "massive" electrons nor "exotic" nuclear reactions to explain the apparent "cold" fusion d-d neutron production results. Instead, it is possible that high voltage electrostatic fields, known to be associated with cracking, can reside across a crack gap long enough for the deuterons to be accelerated to sufficiently high energy to produce the d-d reactions. Interestingly, the electrostatic acceleration is quite similar to that of laboratory accelerators except for its submicron scale. Clearly, much work is still required to determine whether such a crack-generated acceleration mechanism, a "quasi-particle" mechanism, some combination of these, or some other, as yet unidentified mechanism is responsible for the nuclear effects seen in "cold" fusion experiments.

\section{REFERENCES}

1. M. Fleischmann and S. Pons (1989).J. Electmanal. Chem., 261, 301.

2. S. E. Jones, E. P. Palmer, J. B. Czirr, D. L. Decker, G. L. Jensen, T. M. Thorne, and S. F. Taylor (1989). Nature 338, 737.

3. G. Heinicke, (1964). Tribochemistry (Akademic-Verlag, Berlin; V. A. Klyuev, A. G. Lipson, Yu. P. Toporov, A. D. Aliev, A E. Chalykh, and B. V. Deryagin (1984). JETP Phys. Chem. M., $279,1027$.

4. J. T. Dickinson, E. E. Donaldson, and M. K. Park (1981). J. Mater. Sci. 16, 2897.

5. V. A. Klyuev, A. G. Lipson, Yu. P. Toporov, B. V. Deryagin, V. I. Lushchikov, A. V. Strelkov, and E. P. Shabalin, (1986). Sov. Tech. Phys. Lett. 12, 551. 
6. D. L. Book (1981). Physics Vade Mecum (A. I. P., New York), p. 37.

7. W. M. Mueller, J. P. Blackledge, and G. G. Libowitz, eds. (1968). Metal Hydrides (Academic Press, New York), p. 644.

8. H. C. Jamieson et al., (1976). J. Less Common Metals, 50, 85.

9. R. Ryan, M. Fowler, E. Garcia, H. Menlove, M. Miller, A. Mayer, J. Wilhelmy, C. Orth, S. Schmidt, R. Mills, D. Moore, and J. T. Dickinson. Abstracts Selected for Poster Sessions, Workshop on Cold Fusion Phenomena.

10. H. O. Menlove, M. M. Fowler, E. Garcia, A. Mayer, M. C. Miller, R. R. Ryan, and S. E. Jones. Agenda-Workshop on Cold Fusion Phenomena.

11. R. Ryan (private communication). 